Gastroenterologe 2016 $11: 443$

DOI 10.1007/s11377-016-0115-3

Online publiziert: 20 . Oktober 2016

๑) Springer-Verlag Berlin Heidelberg 2016

CrossMark

\section{Roland M. Schmid ${ }^{1}$. Christian Trautwein ${ }^{2}$}

${ }^{1}$ Klinik und Poliklinik für Innere Medizin II: Gastroenterologie, Endokrinologie, Diabetologie, Infektiologie, Klinikum rechts der Isar, Technische Universität München, München, Deutschland

${ }^{2}$ Klinik für Gastroenterologie, Stoffwechselerkrankungen, und Internistische Intensivmedizin (Med. Klinik III), Universitätsklinikum Aachen, Aachen, Deutschland

\title{
Update kolorektales Karzinom
}

Das kolorektale Karzinom ist weltweit an zweiter bzw. bei Männern an dritter Stelle der krebsbedingten Todesursache. Die Prognose des Kolonkarzinoms verbessert sich seit Beginn der 1990er-Jahre. Die 5-Jahres-Überlebensrate für Patienten mit einem kolorektalen Karzinom liegt derzeit bei $64 \%$. Das Kolonkarzinom ist biologisch heterogen. Prof. Kolligs stellt das evidenzbasierte und individualisierte Vorgehen zur Vorsorge vor. Die adjuvante Chemotherapie des Kolonkarzinoms wird im Stadium III generell empfohlen, im Stadium II ist ihr Nutzen fraglich. Für die Zukunft könnte die Tumorlokalisation entscheidend sein, wie Herr Prof. Algül ausführt. Herr Dr. Pohl und Herr Prof. Schmiegel erläutern die medikamentöse Therapie des metastasierten kolorektalen Karzinoms und die durch neue Chemotherapeutika und biologische Substanzen im letzten Jahrzehnt deutlich verbesserte Prognose.

\section{》) Die Prognose des \\ Kolonkarzinoms verbessert sich seit Beginn der 1990er-Jahre}

Zukünftig könnte die Einteilung in molekulare Subgruppen für die Therapie wichtig werden. Die chirurgische Resektion primär resektabler Lebermetastasen ist Standard und ermöglicht grundsätzlich eine Heilung in über $30 \%$ der Fälle. Prof. Lüdde und Kollegen führen die Datenlage zur primären und sekundären Resektabilität von Lebermetastasen aus. Lokalund regional-ablative Verfahren nehmen in der multimodalen Therapie zu, u. a. auch in frühen Linien. Patienten mit kolorektalen Lebermetastasen sollten daher nicht nur zu Beginn, sondern regelmäBig auch im Verlauf in einer interdiszipli- nären Tumorkonferenz zwingend vorgestellt werden. Dr. Ettrich und Prof. Seufferlein erläutern die aktuelle Diagnostik und Therapie des Rektumkarzinoms. Deutliche Unterschiede zwischen Rektum- und Kolonkarzinom zeigen sich indes im Bereich der Anforderungen an die Ausbreitungsdiagnostik sowie der lokalisationsabhängigen perioperativen Therapie und im Bereich der adjuvanten Therapie.

Wir wünschen Ihnen beim Lesen der Artikel viel Spaß und hoffen, Ihnen damit einen aktuellen Stand über die Therapie des kolorektalen Karzinoms vermitteln zu können.

Mit herzlichen Grüßen

Ihre

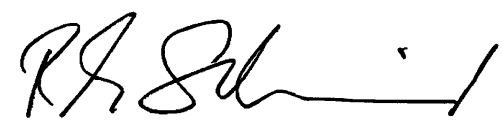

Prof. Dr. R.M. Schmid

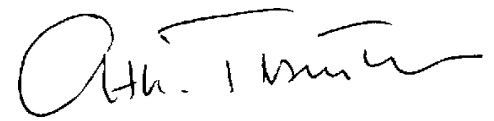

Prof. Dr. Chr. Trautwein

\section{Korrespondenzadresse}

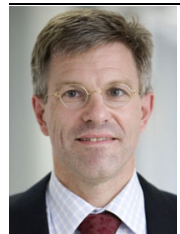

Prof. Dr. R. M. Schmid

Klinik und Poliklinik für Innere Medizin II: Gastroenterologie, Endokrinologie, Diabetologie, Infektiologie, Klinikum rechts der Isar, Technische Universität München Ismaninger Straße 22, 81675 München, Deutschland rolandm.schmid@mri.tum.de

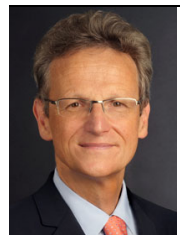

Prof. Dr. C. Trautwein

Klinik für Gastroenterologie, Stoffwechselerkrankungen, und Internistische Intensivmedizin (Med. Klinik III), Universitätsklinikum Aachen Pauwelsstraße 30, 52074 Aachen, Deutschland ctrautwein@ukaachen.de

Interessenkonflikt. R.M. Schmid und C. Trautwein geben an, dass kein Interessenkonflikt besteht. 